\title{
Inspection Robots in Oil and Gas Industry : a Review of Current Solutions and Future Trends
}

\author{
Leijian $\mathrm{Yu}^{1}$, Erfu Yang ${ }^{1 *}$, Peng Ren ${ }^{2}$, Cai Luo ${ }^{3}$, Gordon Dobie ${ }^{4}$, Dongbing $\mathrm{Gu}^{5}$, Xiutian Yan $^{1}$ \\ ${ }^{1}$ Department of Design, Manufacture and Engineering \\ Management, \\ University of Strathclyde, \\ Glasgow G1 1XJ, Scotland, UK \\ *Corresponding author: erfu.yang@strath.ac.uk \\ ${ }^{2}$ College of Information and Control Engineering, \\ China University of Petroleum (East China), \\ Qingdao 266580, China \\ ${ }^{3}$ College of Mechanical and Electronic Engineering, \\ China University of Petroleum (East China), \\ Qingdao 266580, China \\ ${ }^{4}$ Department of Electronic and Electrical Engineering, \\ University of Strathclyde, \\ Glasgow G1 1RD, Scotland, UK \\ ${ }^{5}$ School of Computer Science and Electronic Engineering, \\ University of Essex, \\ Colchester C04 3SQ, UK
}

\begin{abstract}
With the increasing demands for energy, oil and gas companies have a demand to improve their efficiency, productivity and safety. Any potential corrosions and cracks on their production, storage or transportation facilities could cause disasters to both human society and the natural environment. Since many oil and gas assets are located in the extreme environment, there is an ongoing demand for robots to perform inspection tasks, which will be more cost-effective and safer. This paper provides a state of art review of inspection robots used in the oil and gas industry which including remotely operated vehicles (ROVs), autonomous underwater vehicles (AUVs), unmanned ground vehicles (UGVs) and unmanned aerial vehicles (UAVs). Different kinds of inspection robots are designed for inspecting different asset structures. The outcome of the review suggests that the reliable autonomous inspection UAVs and AUVs will gain interest among these robots and reliable autonomous localisation, environment mapping, intelligent control strategies, path planning and Non-Destructive Testing (NDT) technology will be the primary areas of research.
\end{abstract}

Keywords-inspection robots, oil and gas industry, autonomous inspection, defects detection, asset integrity

\section{INTRODUCTION}

The Oil and Gas sector is an underpins modern society. In the 2018 Energy Outlook, British Petroleum (BP) predicted that the absolute consumption of oil and gas would have steady growth to 2040 [1]. The facilities used in oil and gas industry for exploration, production, storage, transportation and distribution are expensive and important assets. If they are not well maintained, their failures may cause catastrophic consequences for both the natural environment and human society. In the Gulf of Mexico, the Deepwater Horizon drilling explosion killed more than 600,000 animals [2]. The Amuay refinery blast injured more than 80 people and damaged several buildings near the plant [3]. Therefore, regular and precise inspection is necessary not only for production but also for safety. However, due to the hazardous environment, it is difficult or expensive to accomplish by human inspectors.

For these reasons, the inspection robots are attractive to the industry. Fig. 1 from [4] shows that the demand of the inspection robots will steadily increase in the foreseeable future. Based on vehicle type, the market of inspection robots in oil and gas industry can be divided into the following categories: Remotely operated vehicles (ROVs), Autonomous underwater vehicles (AUVs), Unmanned ground vehicles (UGVs) and Unmanned aerial vehicles (UAVs) [5]. These robots have different mechanisms and structures for different inspection tasks. Some of them are focused on inspecting oil storage tanks, while some of them are designed for pipeline inspection. Nevertheless, most of them need experienced engineers to manipulate them to conduct the inspection process. Greater levels of robot's flexibility and autonomy can make the inspection more intelligent and efficient. To have a comprehensive understanding inspection robot in oil and gas industry, this paper focuses on reviewing the key technologies in different kinds of inspection robots, discussing the challenges and highlighting the trends in future research, which will make the inspection process more efficient, intelligent and cost-effective.

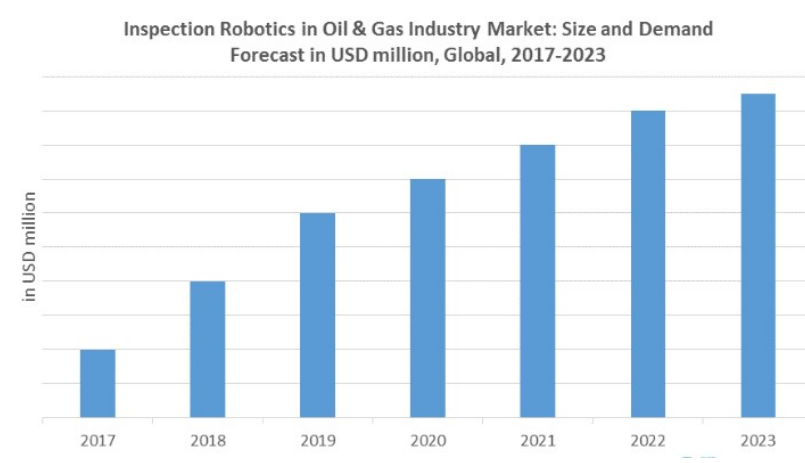

Figure 1. Inspection robots in oil and gas industry [4].

As the ROVs and AUVs are both underwater unmanned vehicles (UUVs), they will be covered in the related sections UUVs in this paper. The rest of the paper is organised as follows. In Section II, the common sensors used for inspection robots are presented. while the current inspection robot solutions are introduced in Section III. Then, Section IV discusses the challenges and future trends in high-level flexibility and autonomy of inspection robots. Finally, this paper is concluded in Section $\mathrm{V}$. 


\section{INSPECTION METHODS AND SENSORS}

For the inspection purposes in oil and gas industry, a number of onboard NDT sensors are used to detect corrosions and cracks. According to different types of sensors, the most commonly applied inspection technologies can be roughly divided into four classes, i.e., visual inspection, ultrasonic inspection, magnetic inspection and eddy current inspection.

Visual inspection is one of the oldest and popular inspection means. At the very beginning, experienced engineers used their naked eye to check the condition of assets. But now, cameras have allowed the inspection robots have the ability to pursue a view of the structure. When in visual degradation environment, LEDs can be used to deal with the absence of proper illumination. Visual inspection is simple and it is one of the easiest inspection techniques to perform. However, it is only suitable for detecting damages on the surface and the inspection quality is sensitive to illumination [6].

Besides the visual inspection mentioned previously, the ultrasonic sensor is another primary sensor found on many inspection robots. Ultrasonic sensors can emit and receive the ultrasound, which is propagated into the material. Cracks can be detected by measuring the time difference between the generated and reflected ultrasound. The comparison of these two signals tells the location and size of the crack. There are many advantages in using ultrasonic sensors, such as high accuracy, high sensitivity and suitability for monitoring all kinds of materials. However, it will not work when the defect lays along the line of wave travelling [7].

Magnetic sensitive sensors work with ferrous material assets. After applying a magnetic field on these facilities, most of the magnetic flux lines will go through these metal materials. If there is a defect, magnetic flux lines will be bent. Some of the magnetic flux lines will leak out. The magnet sensitive sensors can detect the magnetic leakage field. The detected signal can be analysed to reveal the changes in structure. This method can realise relatively high-speed inspection and very good sensitivity to pitting. However, it can only work for ferromagnetic materials and the sizes of defects detected are very limited [8].

Eddy current inspection is similar to magnet sensitive inspection in some ways. It uses eddy currents generated by coils. When there is a crack in the structure, the eddy current will be altered. At the same time, the impedance of the coil will also be affected. Monitoring the change in impedance in the coils can tell the condition of the facility. This method is sensitive to surface detects and can be used to inspect multilayer structures. Nonetheless, it is very susceptible to magnetic permeability changes and cannot detect the defects parallel to the surface [9].

Through the sensor technologies discussed above, we can see that each sensor has its own advantages and disadvantages. When choosing the proper sensor for inspection robots, the application scenario and constraints need be taken into consideration.

\section{CURRENT InSPECTION Robots REVIEW}

\section{A. Unmanned Ground Vehicles}

According to the shape and function of the onshore facilities in oil and gas industries, the equipment can be roughly divided into vertical structures and pipelines. The vertical structures contain drilling, production and storage assets, such as flare stacks and tanks. Pipelines are mostly focused on transportation purpose. To inspect these kinds of facilities, UGVs are the most popular choice nowadays.

\section{1) Vertical Structure Inspection}

For inspecting the vertical structures, wall climbing robots have gained great interests. The climbing technologies are the main difference between these robots. At the same time, the most important task in the design and development of a climbing robot is to develop an appropriate mechanism to ensure that the robot adheres to different types of walls and surfaces reliably without sacrificing its mobility. According to the adhesion and locomotion principles, the climbing methods can be cauterised as magnetic adhesion mechanism, pneumatic adhesion mechanism and bio-inspired grasping grippers [10], as shown in Fig. 2.

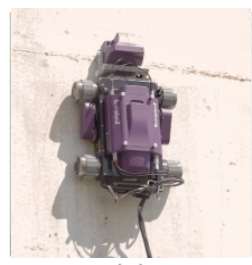

(a)

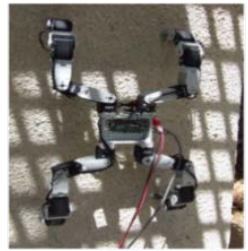

(c)

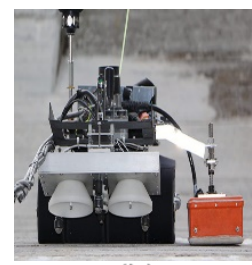

(b)

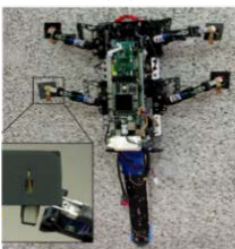

(d)
Figure 2. (a) a magnetic adhesion robot [11], (b) a pneumatic adhesion robot [14], (c) a cat insperied adhesive robot [15], (d) a gecko inspired robot [16].

Vertical structures in the oil and gas company are usually made of carbon steel. Since this kind of material is ferromagnetic, magnetic adhesion can be highly desirable in this kind environment. There is a lot of work utilising magnetic adhesion to inspect these facilities. One method is to use permanent magnets. Such as [11], they select strength permanent magnets as the adhesion mechanism, which can hold the robot firmly on the walls. Another advantage of these methods is robots do not need extra power for the adhesion mechanism. In some circumstances, variable adhesion is required and the speed of switching required is high, the electromagnetic adhesion mechanism will become specifically useful [12].

Pneumatic adhesion mechanism is another widely used technology in vertical structure inspection robots. The attraction force between the robot and the wall is proportional to the pressure difference between the pressure chamber or suction cups and the atmosphere. Unlike magnetic adhesion methods which can only work 
on the ferromagnetic surfaces, pneumatic adhesion mechanism is suitable for a wider range of materials. Using suction cups is a very popular method. In [13], three suction cups, a supporting plate, a vacuum pump, and some accessories were used to compose the suction module. The ICM Rover equips vacuum adhesion mechanism as fall protector, while performing inspection tasks [14].

There is also a variety of works adopting biomimetic adhesion methods to realise the excellent climbing robots. In [15], researchers developed a robot, which consists of four legs with gripping devices made of 12 fishing hooks. This robot can imitate the movement of rock-climbers and the way cats hold surfaces when they climb on vertical direction. A gecko-inspired adhesives method was proposed in [16]. Being inspired by the gecko toes, it used a rigid tile supported by a compliant material and loaded by an inextensible tendon. This mechanism allowed the climbing part to make a full contact with the surfaces.

\section{2) Onshore Pipeline Inspection}

For onshore pipeline inspection robots, the style of locomotion is a vital part, which can reflect the whole performance of the robots [17]. According to the difference of driving source and control ability of movement mechanism, robots can be sorted into pig, wheeled, tracks, legged, inchworm, snake and screw type [18]. Fig.3 gives some examples about these kinds of robots.

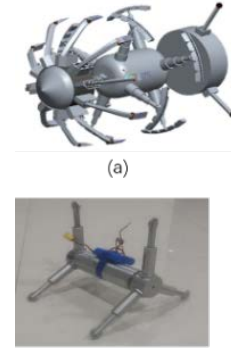

(d)

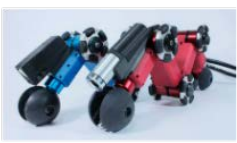

(b)

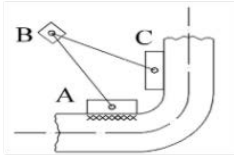

(e)

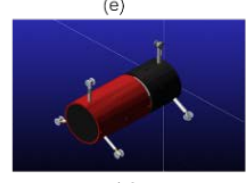

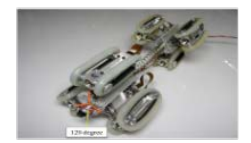

(c)

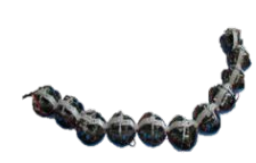

(f) front or back module. Compared with other types of inspection robots, it has an advantage in curved pips. Snake type [24] consists of several identical body segments with joints, which allows it to generate a wide range of different motions. The screw type [25] moves forward through the rotary motion, achieving a good performance in vertical pipelines.

\section{B. Unmanned Underwater Vehicles}

Oil and gas companies have thousands of kilometres of pipelines and other assets in the sea to produce and transport their products. These undersea structures can easily develop corrosion and cracks since these structures are often made from metal materials. In order to prevent financial and environmental disasters caused by leaking out of the product, these facilities need to be inspected frequently. ROVs and AUVs are effective and affordable platforms to perform underwater inspection tasks. The inspection ROVs are operated by the surface operator. They are alternative vehicles to human workers in conditions that are too deep and too dangerous for human beings. What is more, with the help of ROVs, inspection tasks can be performed 24 hours and 7 days. Due to these advantages, the oil and gas technologies have developed ROV technologies from the 1980s [26]. After that, a large amount of advanced ROVs with a group of sensors were used to carry out inspection tasks which vary in size and weight [27][28][29][30][31]. These ROVs consist of a vehicle body, control cabin, umbilical, video cameras, handling system, launch system and power supplies. The main features of these ROVs are summarized in TABLE I.

\begin{tabular}{|c|c|c|c|c|}
\hline $\begin{array}{r}\text { TAB } \\
\text { ROVs }\end{array}$ & $\begin{array}{c}\text { Max } \\
\text { Depth } \\
\text { (meters) }\end{array}$ & $\begin{array}{c}\text { Weight } \\
\text { (kg) }\end{array}$ & $\begin{array}{c}\text { VS COMP } \\
\text { Forward } \\
\text { Speed } \\
\text { (knots) }\end{array}$ & $\begin{array}{l}\text { SION } \\
\begin{array}{l}\text { Size } \\
(\mathbf{m m})\end{array}\end{array}$ \\
\hline $\begin{array}{c}\text { VideoRay } \\
\text { PRO } 4\end{array}$ & 305 & 38.5 & 4.2 & $375,290,220$ \\
\hline $\begin{array}{c}\text { Novaray } \\
\text { model } \\
2000\end{array}$ & 305 & 25.5 & 6 & $1020,997,229$ \\
\hline FALCON & 300 & 60 & 3 & $1000,500,600$ \\
\hline $\begin{array}{c}\text { CONSTR } \\
\text { UCTOR } \\
220 \mathrm{HP} \\
\end{array}$ & 3048 & 4500 & 3.1 & $\begin{array}{c}3220,1700 \\
2165\end{array}$ \\
\hline Mojave & 300 & 85 & 3.5 & $1000,600,500$ \\
\hline
\end{tabular}

For the deep-sea inspection tasks, the major cost of using an ROV is caused by the human operator. If these tasks can be performed autonomously, this cost can be reduced. Based on the previous technologies and economic constraints, using the AUVs may be a replacement for the ROVs for inspection purpose and currently it has gained great research interest. Compared with ROVs, AUVs demand more accurate and efficient sensors, guidance systems and algorithms for the execution of this kind of mission. The LIRMM [32] presented a Deformable Virtual Zones (DVZ) method that is a sensor-based control approach. It builds the model of a virtual zone around the robot. Once the obstacles are detected by proximity sensors, there will be deformation and the control signal will be calculated by minimising the deformation in the DVZ. The FlatFish project [33] utilized the asset layoutbased navigation methods. Since almost all of the underwater assets are connected together, they can be inspected by following pipelines and tie-backs to reach the 
different part of facilities. The sonar and camera are used to target and inspect the facilities. In [34], image-processing technologies are adopted to AUV's navigation. At first, it detects the pipeline corners in the captured image. After that, the obstacles are identified by Hough Transform (HT) and velocity and angle values can be calculated according to this information. Finally, the AUV can move along the pipeline by itself and inspect these structures autonomously.

\section{Unmanned Aerial Vehicles}

UAVs equipped with relative sensors can work as an excellent alternative to traditional inspection techniques. It can not only save time but also lower the cost. The North Sea E\&P company conducted a survey and showed that using UAVs to inspect assets can be twenty times faster and half the cost of traditional inspection methods [35]. This kind of method has gained great interest in inspecting assets in oil and gas industry. Until now, all of the commercial inspection UAVs are manually controlled. During the inspection procedure, the UAV will be controlled by one experienced pilot, while the live video for inspection purpose is monitored by another experienced inspection engineer. Famous oil and gas companies in the world such as BP, Shell, Apache, BG Group and Statoil have cooperated with Cyberhawk, which is the world leader in drone inspection to inspect their facilities. Intel Falcon 8 plus used by Cyberhawk has shown a reliable and efficient performance [36]. The Intel Falcon 8 plus has a patented V-shaped design with eight rotors, which makes the UAV more stable and ensures unobstructed data capture procedure. By carrying three redundant inertial measurement units (IMUs) with efficient data fusion technology, the flying system can perform reliable responsiveness and stability during flights. The inspection module consists of an RGB camera and a thermal camera, which helps UAV navigate while capturing detailed data for orthography and 3D reconstruction that can be used for inspecting the assets and further analysation [37]. Another notable inspection UAV is ELIOS, the first collision-tolerant drone developed by Flyability. This UAV is surrounded by a carbon fibre shell, which can protect the body from the collision. When collision to the obstacles, the UAV will bounce off and roll along the surface to find the path. What is more, it can finish the inspection task in any light conditions with the help of the HD camera, thermal camera and an LED lighting system.

For UAV inspection, one UAV needs two experienced human engineers. If the inspection tasks can be performed autonomously, the inspection procedure can not only be speeded up but also improve cost efficiency by reducing engineering labor fees and minimising the accidents due to human operate errors. The Petroleum Institute of Abu Dhabi has developed a UAV autonomous tracking and navigation controller for inspecting straight oil and gas pipelines [38]. Their autonomous procedure contains four parts: Firstly, the gradient of Gaussian is used to extract the edge of the object in image sequences. Secondly, the HT is implied to identify the pipeline. After that, a PID controller is designed to achieve angular and lateral correction, which ensures the position and orientation of the UAV is aligned with the pipeline. Finally, automated navigation along the pipeline is realised.

\section{Challenges AND RESEARCH DiRECTIONS}

From the survey above, it can be concluded that robots play an important role for inspection purpose in oil and gas industries. In these robots, UAV systems can be efficient alternatives for achieving the same goals to UGVs. AUVs outperform ROVs in terms of efficiency and cost in underwater inspection tasks. The autonomous inspection UAVs and AUVs do not need human interaction, which can further reduce the operational cost and timeconsuming. Meanwhile, the autonomous inspection systems have become the main development direction of energy industries [39]. For autonomous inspection robots, the capabilities of autonomous localisation, navigation and inspection play crucial roles. UAVs and AUVs are both 6 Degree of freedoms (DoFs) robots, and they both adopt visual inspection technologies. The same development frame can be implied to both of them. What is more, some hardware and software can work both for UAVs and AUVs, such as px4 open source autopilot [40]. Approaches discussed here are suitable for both UAVs and AUVs. According to the survey above, the autonomous inspection framework can be concluded as Fig.4. It consists of Visual Simultaneous Localisation and Mapping (VSLAM), the control part, path planning and defect detection.

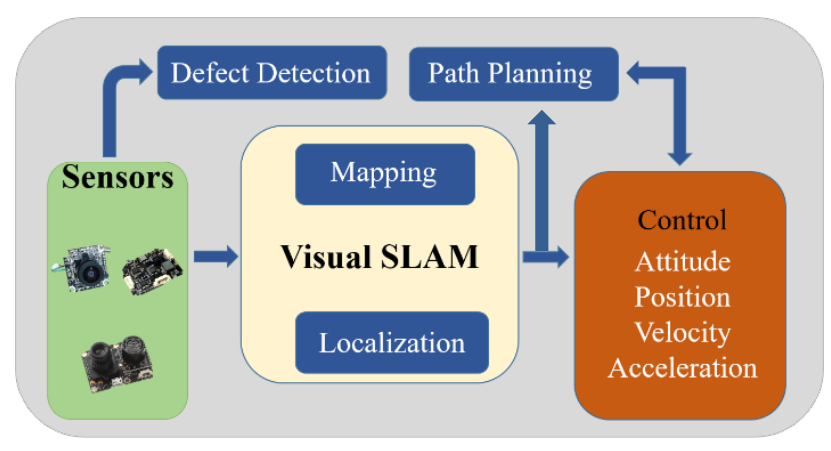

Figure 4. Framework of autonomous inspection

\section{A. Autonomous Localisation and Mapping}

Autonomous localisation and environment mapping are the basic requirements for navigation. Robots need to know where it is before planning the path. However, in the assets inspection scenario, the electromagnetically guided localisation systems are constrained. As UAVs and AUVs both utilise vision information to inspect the structure. Vision-based localisation and navigation systems have become popular these days. They are based on the images captured by the onboard camera, and VSLAM algorithms can locate robots' position, estimate its state and simultaneously build the map of the surrounding environment. These features make it suitable for visual guide systems. Some related works have proved the reality of these methods. Graph-based SLAM has been deployed in UAVs and AUVs to realise the robot localisation [41]. There is no doubt that the previously introduced methods have made a great improvement in intelligent and autonomous 6-DoF robots. However, these approaches are still in the start-up stage. Unlike UGVs, UAVs and AUVs cannot stop operating while it in the uncertain state. It 
means that VSLAM should be able to locate the position and respond to the dynamic environment quickly. For the inspection tasks, the robots should be close to the facilities, which will cause the scenery changes very fast. In other words, perceiving the dynamic environment is a significant problem that needs to be solved. The tolerance of image blur, object occlusions and illumination changes should also be taken into consideration.

\section{B. Intelligent Control Strategies and Path Planning}

Another challenge is to integrate the VSLAM with intelligent control strategies and path planning to realise high-level reliable and flexible navigation. Intelligent navigation methods can make the inspection progress more efficient and safer. This survey also indicates that visual localisation systems are not fully integrated with the navigation systems. The previously presented works can only work under specific assumptions, such as the pipelines are straight or some parameters are known [34][38]. In other words, their works can just be a kind of simulation for the autonomous efficient inspection. It is clear that UAVs and AUVs are more efficient than other robots which heavily relied on their agile maneuvering capabilities. Therefore, it is essential to pay more attention to it and make intelligent control strategies and path planning a further step.

\section{Inspection Technologies}

It can be clearly seen from the review that one experienced engineer is needed to monitor the image sequences captured by the UAVs and AUVs. What is more, to realise a fully autonomous inspection system, autonomous detecting corrosions and cracks is essential. With the development of image recognition, especially with the deep learning algorithms, it is possible to inspect the assets in oil and gas industries autonomously. Deep learning methods, especially convolutional neural networks, which has made many breakthroughs in computer vision tasks [42][43][44]. It can extract features autonomously for all kinds of objects, which is more accurate and robust than traditional man designed features, such as SIFT [45] and HOG [46]. It has led to great improvements in many vision inspection tasks. Beijing University of Science and Technology has adopted the deep learning methods to detect steel surface, which is vital to the steel product. With the help of deep learning method, the detection precision of hot rolled plates has been improved around 16\% than traditional methods [47]. In [48], a deep learning algorithm was used to detect breaches, dents, burrs and abrasions on the sealing surface of a container. The accuracy and efficiency of this method has been proved by the real-world test in the filling line. The autonomous detection of concrete structure cracks can also be realised by deep learning. Dung C. V. developed a deep learning based autonomous concrete crack detection algorithm, which can reach approximately $90 \%$ of the maximum Average precision (AP) and F-Score (F1) on validation and test datasets [49]. With so many successful works in inspection tasks with deep learning, it is possible to develop autonomous corrosion and cracks detection approaches in the future. As a result, the inspection quality, speed, as well as the overall inspection process will be improved.

\section{CONCLUSION}

In this paper, four kinds of inspection robots in oil and gas industries have been surveyed. Among these robots, autonomous UAVs are efficient alternatives to UGVs. At the same time, AUVs can be more efficient than ROVs. The autonomous UAVs and AUVs will be the research hot pot in oil and gas industries. What is more, with the development of VSLAM, intelligent control strategies and path planning methods, the autonomous localisation and navigation performance of UAVs and AUVs still can be further improved. The autonomous inspection will also come true with the help of advanced computer vision algorithms, especially with the deep learning method. The authors believe that the maturity level of autonomous inspection UAVs and AUVs will be developed, considering the robustness and reliability.

\section{ACKNOWLEDGMENT}

This work is supported in part by the UK Oil and Gas Technology Centre (OGTC) under the LOCUST research project (2019-2021, Grant No.: AI-P-028). Mr Leijian Yu is funded by the China Scholar Council and the International Fees Only Studentship from the University of Strathclyde (2018-2021). The authors would also like to thank the OGTC robotics team at the University of Strathclyde for their kindly support.

\section{REFERENCES}

[1] "Overview | Energy economics | Home", BP global, 2019. [Online].Available:https://www.bp.com/en/global/corporat e/energy-economics/energy- outlook/ introduction/overvie w.html.

[2] "Gulf oil leak: Biggest ever, but how bad?", BBC News, 2019. [Online]. Available: https: //www.bbc.co.uk/ news/sc ience-environment-10851837.

[3] "Blast halts Venezuela refinery", BBC News, 2019. [Online]. Available: https://www.bbc.co.uk/news/worldlatin-america-19378657.

[4] "Inspection Robotics in Oil \& Gas Industry Market | Growth, Trends, and Forecast (2018 - 2023)", Mordorintelligence.com, 2019. [Online]. Available: https://www.mordor intelligence.com/industry-reports/inspection-robotics-in-oi l-and-gas-industry-market.

[5] R. ltd, "Inspection Robotics in Oil \& Gas Industry: Global Market 2017-2025 by Robot Type, Sub-system and Region", Researchandmarkets.com, 2019. [Online]. Available: https://www.researchandmarkets.com/research/4dvv8 g/inspection.

[6] S. Hajjaj and I. Khalid, "Design and Development of an Inspection Robot for Oil and Gas Applications", International Journal of Engineering \& Technology, vol. 7, no. 435, p. 5, 2018.

[7] P. Shull, Nondestructive evaluation : theory, techniques, and applications. CRC press, 2016.

[8] Y. Shi, C. Zhang, R. Li, M. Cai and G. Jia, "Theory and Application of Magnetic Flux Leakage Pipeline Detection", Sensors, vol. 15, no. 12, pp. 31036-31055, 2015.

[9] M S Safizadeh, M. Hasanian, "Gas pipeline corrosion mapping using pulsed eddy current technique", International Journal of Advanced Design and Manufacturing Technology, vol. 5, no. 1, pp. 11-18, 2011.

[10] M. F. Silva, J. A. T. Machado, and J. K. Tar, "A Survey of Technologies for Climbing Robots Adhesion to Surfaces," in 2008 IEEE International Conference on Computational Cybernetics, 2008, pp. 127-132. 
[11] "Scorpion2 and Swift Remote-Access Tank Shell Inspection Solution", Eddyfi, 2019. [Online]. Available: https://www.s ilverwingndt.com/scorpion2/.

[12] M. F. Silva, R. S. Barbosa, and A. L. C. Oliveira, "Climbing Robot for Ferromagnetic Surfaces with Dynamic Adjustment of the Adhesion System,” J. Robot., vol. 2012, pp. 1-16, Mar. 2012.

[13] Y. Guan et al., “A Modular Biped Wall-Climbing Robot With High Mobility and Manipulating Function," IEEE/ASME Trans. Mechatronics, vol. 18, no. 6, pp. 17871798, Dec. 2013.

[14] "ICM Rover - Advanced Vacuum Adhesion Inspection Crawler", Robotics.innotecuk.com, 2019. [Online]. Available: http://robotics.innotecuk.com/icm-rover-advanced-vac uum-adhesion-inspection-crawler/.

[15] A. Sintov, T. Avramovich, and A. Shapiro, "Design and motion planning of an autonomous climbing robot with claws,” Rob. Auton. Syst., vol. 59, no. 11, pp. 1008-1019, Nov. 2011.

[16] E. W. Hawkes, E. V. Eason, A. T. Asbeck, and M. R. Cutkosky, "The Gecko's Toe: Scaling Directional Adhesives for Climbing Applications,” IEEE/ASME Trans. Mechatronics, vol. 18, no. 2, pp. 518-526, Apr. 2013.

[17] Wang, D.J., Li, R.P. and HUANG, G.M., "Progresses in Study of Pipeline Robot," in 2012 Proceedings of International Conference on Modelling, Identification and Control, Sep, 2012.

[18] J. M. Mirats Tur and W. Garthwaite, "Robotic devices for water main in-pipe inspection: A survey,” J. F. Robot., vol. 27, no. 4, pp. 491-508, Jun. 2010.

[19] A. A. Mazreah, F. B. I. Alnaimi, and K. S. M. Sahari, "Novel design for PIG to eliminate the effect of hydraulic transients in oil and gas pipelines,” J. Pet. Sci. Eng., vol. 156, pp. 250 257, Jul. 2017.

[20] A. Kakogawa and S. Ma, "Design of a multilink-articulated wheeled pipeline inspection robot using only passive elastic joints,” Adv. Robot., vol. 32, no. 1, pp. 37-50, Jan. 2018.

[21] Y.-S. Kwon and B.-J. Yi, "Design and Motion Planning of a Two-Module Collaborative Indoor Pipeline Inspection Robot,” IEEE Trans. Robot., vol. 28, no. 3, pp. 681-696, Jun. 2012.

[22] A. A. Gargade and S. S. Ohol, "Development of Actively Steerable In-pipe Inspection Robot for Various Sizes," in Proceedings of the Advances in Robotics on - AIR '17, 2017, pp. 1-5.

[23] C. Nițu, B. Grămescu, A. S. Hashim, and M. Avram, "Inchworm Locomotion of an External Pipe Inspection and Monitoring Robot,” Springer, Cham, 2019, pp. 464-470.

[24] F. Trebuňa, I. Virgala, M. Pástor, T. Lipták, and L. Miková, "An inspection of pipe by snake robot," Int. J. Adv. Robot. Syst., vol. 13, no. 5, Sep. 2016.

[25] H. Tourajizadeh, M. Rezaei, and A. H. Sedigh, "Optimal Control of Screw In-pipe Inspection Robot with Controllable Pitch Rate,” J. Intell. Robot. Syst., vol. 90, no. 3-4, pp. 269-286, Jun. 2018.

[26] Shukla A, Karki H. Application of robotics in offshore oil and gas industry-A review Part II[J]. Robotics and Autonomous Systems, 2016, 75: 508-524.

[27] "VideoRay ROVs - Underwater Remotely Operated Vehicles", Videoray.com, 2019. [Online]. Available: http://www. Videoray.com/.

[28] "Nova Ray® Subsea Systems ROV (Remotely Operated Vehicle) The Current ExpertsTM", Novaray.com, 2019. [Online]. Available: http://www.novaray.com/.

[29] "Electric Underwater Robotics (ROVs) | Saab Seaeye", Saabseaeye.com, 2019. [Online]. Available: https://www.sa abseaeye.com.

[30] "Underwater Drones (ROV, USV) for sale in California Deep Ocean Engineering", Deepocean.com, 2019. [Online]. Available: https://www.deepocean.com/.
[31] "Forum Energy Technologies", F-e-t.com, 2019. [Online]. Available: https://www.f-e-t.com.

[32] P. K. Paim, B. Jouvencel, and L. Lapierre, "A Reactive Control Approach for Pipeline Inspection with an AUV," in Proceedings of OCEANS 2005 MTS/IEEE, pp. 1-6.

[33] J. Albiez et al., "Repeated close-distance visual inspections with an AUV," in OCEANS 2016 MTS/IEEE Monterey, 2016, pp. 1-8.

[34] C. Wang and L. Cui, "The Implementation of Automatic Inspection Algorithm for Underwater Vehicles Based on Hough Transform,” in Proceedings of the 2018 7th International Conference on Sustainable Energy and Environment Engineering (ICSEEE 2018), 2019.

[35] "Drones halve the cost of inspection, Cyberhawk says", Offshore Energy Today, 2019. [Online]. Available: https://www.offshoreenergytoday.com/drone-inspectionshalve-the-cost-of-inspection-cyberhawk/.

[36] "Intel and Cyberhawk Inspect Gas Terminal through Lens of Commercial Drone Technology - Cyberhawk", Cyberhawk, 2019. [Online]. Available: https:// thecyberhaw k.com/intel-cyberhawk-inspect-gas-terminal-lens-commerc ial-drone-technology/.

[37] I. System, "Intel ${ }^{\circledR}$ Falcon ${ }^{\mathrm{TM}}$ 8+ System", Intel, 2019. [Online]. Available: https://www.intel.co.uk/content/www/ uk/en/products/drones/falcon-8.html.

[38] A. Shukla, H. Xiaoqian, and H. Karki, "Autonomous tracking and navigation controller for an unmanned aerial vehicle based on visual data for inspection of oil and gas pipelines," in 2016 16th International Conference on Control, Automation and Systems (ICCAS), 2016, pp. 194 200.

[39] V. Sudevan, A. Shukla, and H. Karki, "Inspection of Vertical Structures in Oil and Gas Industry: A Review of Current Scenario and Future Trends,” pp. 65-68, Jul. 2018.

[40] "Open Source for Drones - PX4 Open Source Autopilot", PX4 Open Source Autopilot, 2019. [Online]. Available: https://px4.io/.

[41] A. Annaiyan, M. A. Olivares-Mendez, and H. Voos, "Realtime graph-based SLAM in unknown environments using a small UAV," in 2017 International Conference on Unmanned Aircraft Systems (ICUAS), 2017, pp. 11181123.

[42] F. Gao, F. Ma, J. Wang, J. Sun, E. Yang, and H. Zhou, "Visual Saliency Modeling for River Detection in HighResolution SAR Imagery,” IEEE Access, vol. 6, pp. 10001014, 2018.

[43] F. Gao, T. Huang, J. Sun, J. Wang, A. Hussain, and E. Yang, "A New Algorithm for SAR Image Target Recognition Based on an Improved Deep Convolutional Neural Network,” Cognit. Comput., pp. 1-16, Jun. 2018.

[44] Z. Yue et al., "A Novel Semi-Supervised Convolutional Neural Network Method for Synthetic Aperture Radar Image Recognition,” Cognit. Comput., pp. 1-12, Mar. 2019.

[45] D. G. Lowe, "Distinctive Image Features from ScaleInvariant Keypoints,” Int. J. Comput. Vis., vol. 60, no. 2, pp. 91-110, Nov. 2004.

[46] N. Dalal and B. Triggs, "Histograms of Oriented Gradients for Human Detection," in 2005 IEEE Computer Society Conference on Computer Vision and Pattern Recognition (CVPR'05), vol. 1, pp. 886-893.

[47] H. Di, X. Ke, Z. Peng, and Z. Dongdong, "Surface defect classification of steels with a new semi-supervised learning method,” Opt. Lasers Eng., vol. 117, pp. 40-48, Jun. 2019.

[48] Y. Li et al., "Research on a Surface Defect Detection Algorithm Based on MobileNet-SSD,” Appl. Sci., vol. 8, no. 9, p. 1678, Sep. 2018.

[49] C. V. Dung and L. D. Anh, "Autonomous concrete crack detection using deep fully convolutional neural network," Autom. Constr., vol. 99, pp. 52-58, Mar. 2019. 